\title{
Adaptive Two-Pass Rank Order Filter to Remove Impulse Noise in Highly Corrupted Images
}

\author{
Xiaoyin Xu, Member, IEEE, Eric L. Miller, Senior Member, IEEE, Dongbin Chen, and Mansoor Sarhadi
}

\begin{abstract}
In this paper, we present an adaptive two-pass rank order filter to remove impulse noise in highly corrupted images. When the noise ratio is high, rank order filters, such as the median filter for example, can produce unsatisfactory results. Better results can be obtained by applying the filter twice, which we call two-pass filtering. To further improve the performance, we develop an adaptive two-pass rank order filter. Between the passes of filtering, an adaptive process is used to detect irregularities in the spatial distribution of the estimated impulse noise. The adaptive process then selectively replaces some pixels changed by the first pass of filtering with their original observed pixel values. These pixels are then kept unchanged during the second filtering. In combination, the adaptive process and the second filter eliminate more impulse noise and restore some pixels that are mistakenly altered by the first filtering. As a final result, the reconstructed image maintains a higher degree of fidelity and has a smaller amount of noise. The idea of adaptive two-pass processing can be applied to many rank order filters, such as a center-weighted median filter (CWMF), adaptive CWMF, lower-upper-middle filter, and soft-decision rank-order-mean filter. Results from computer simulations are used to demonstrate the performance of this type of adaptation using a number of basic rank order filters.
\end{abstract}

Index Terms-Center-weighted median filter (CWMF), error index matrix, impulse noise, lower-upper-middle (LUM) filter, median filter, SD-ROM filter, spatial distribution of impulse noise.

\section{INTRODUCTION}

$\mathbf{I}$ $\mathrm{N}$ IMAGE processing, the median filter is usually used to remove impulse noise [1]. Compared with convolutional filters, the median filter is more robust in that a single very unrepresentative pixel in the filter window will not affect the median value significantly. Also, since the median must actually be one of the pixels in the filter window, the median filter does not create new pixel values when the filter crosses an edge. For this reaso, the median filter is better at preserving sharp discontinuities than spatial averaging filters [2]. Unfortunately, the median filter is prone to alter pixels undisturbed by the noise [2], [3], thereby causing a number of artifacts including edge jitter [4], [5] and

Manuscript received October 20, 2002; revised October 13, 2003. This work was supported in part by CenSSIS, the Center for Subsurface Sensing and Imaging Systems, under the Engineering Research Centers Program of the National Science Foundation (NSF) under Award EEC-9986821, by an ARO MURI on Demining under Grant DAAG55-97-1-0013, and by the NSF under Award 0208548. The associate editor coordinating the review of this manuscript and approving it for publication was Dr. Giovanni Ramponi.

X. Xu and E. L. Miller are with the Center for Subsurface Sensing and Imaging Systems, Department of Electrical and Computer Engineering, Northeastern University, Boston, MA 02115 USA (e-mail: xxu@ece.neu.edu; elmiller@ece.neu.edu).

D. Chen and M. Sarhadi are with the Department of Systems Engineering, Brunel University, Uxbridge, Middlesex UB8 3PH, U.K. (e-mail: dongbin. chen@brunel.ac.uk; mansoor.sarhadi@brunel.ac.uk).

Digital Object Identifier 10.1109/TIP.2004.823827 streaking [6]. Modified forms of the median filter which still retain the rank order structure have been proposed to overcome these shortcomings. Basically, the task is to decide when to apply the median filter and when to keep pixels unchanged [7]. Among those are center-weighted median filters (CWMFs) [8]-[11], which give the current pixel a large weight and the final output is chosen between the median and the current pixel value, detail-preserving median filters [12], and rank-ordered mean filter [13] which excludes the current pixel itself from the median filter, progressive switching median filter (PSM) [14], soft-decision-based filter [7], [15] and predication-based filter [16]. A different kind of weighted median filter has been proposed by Yin et al. [17] where a nonnegative integer weight is assigned to each position in the filter window. Recently, impulse noise removal based on fuzzy logic has been attracting research effort [18].

In this paper, we propose a method that improves the performance of the median filter and other rank order filters by analyzing the spatial distribution of the estimated impulse noise. The impulse noise is estimated by subtracting the rank order filter output from the observation. Using an underlying rank order filter, be it a standard median filter, CWMF, soft-decision rank-order-mean filter (SD-ROM) filter or the like, our method looks for irregularities in the spatial distribution of the estimated impulse noise over a subset of the image. The subset can be a column or a row. Once an irregularity is detected, some pixels changed by the first filtering are replaced by their original pixel values and subsequently kept unchanged during the second pass of the filter. Irregularities are detected adaptively in a decision-theoretic framework by scanning the image in a specific direction.

Our method aims to achieve two objectives. First, the algorithm uses two-pass rank order filtering to remove more noise than is normally the case when the noise ratio is high. Second, by exploiting the spatial distribution of the estimated impulse noise the algorithm corrects errors made by the first pass filtering operation. By doing so, improved results are obtained in terms of better visual appreciation and higher peak signal-to-noise ratio (PSNR) again relative to the single-pass, nonadaptive version of these rank order filters.

For concreteness, the details of our method are described in the context of a median filter although: 1) where appropriate, we indicate the minor changes required to employ the technique using any rank order filter and 2) in the examples at the end of the paper, we do in fact validate the performance using a number of such filtering options.

The paper is organized as follows. Section II discusses impulse noise models, the standard median filter, and presents our adaptive, two-pass approach. Section III demonstrates the 
performance of our algorithm using a number of common test images. We also extend the idea of adaptive two-pass filtering to other popular rank order filters and show that improved results are obtained in each case. Finally, conclusions are given in Section IV.

\section{AlgORITHM}

In this section, we start by briefly describing the impulse noise model and the standard median filter. Then we introduce an adaptive two-pass median filtering (ATPMF) process. And it will be made clear that the key part of our method is independent of the underlying impulse noise detection scheme and therefore our method can be generalized to other rank order filters in a straightforward manner.

\section{A. Noise Model}

We define the noise ratio as

$$
\text { Noise ratio }=\frac{\text { Number of pixels of impulse noise }}{\text { Total number of pixels in the image }}(\%) \text {. }
$$

Impulse noise can be described by a joint probability distribution describing the spatial distribution of the impulses as well as their amplitudes. As is typically the case, these two quantities are considered to be independent. Hence, denoting an $M \times N$ matrix $\mathbf{U}$ as the impulse noise, mathematically we can write

$$
\mathbf{U}=\mathbf{U}_{\mathrm{POS}} \odot \mathbf{U}_{\mathrm{AMP}}
$$

where matrix $\mathbf{U}_{\mathrm{POS}}$, a matrix of size $M \times N$, represents the positions of the impulse noise $\mathbf{U}_{\mathrm{AMP}}$, a matrix of size $M \times$ $N$ represents the amplitudes of the impulse noise at each pixel position, and $\odot$ denotes the point-by-point multiplication. For the noise model, we assume that the impulse noise satisfies a binary distribution at each pixel $\mathrm{U}_{\mathrm{POS}}(m, n)$

$$
\begin{aligned}
& \operatorname{Prob}\left\{\mathbf{U}_{\operatorname{POS}}(m, n)=1\right\}=q \\
& \operatorname{Prob}\left\{\mathbf{U}_{\operatorname{POS}}(m, n)=0\right\}=1-q, \\
& \qquad m=1, \ldots, M, \quad n=1, \ldots, N
\end{aligned}
$$

where $0 \leq q \leq 1$. The binary distribution indicates that at position $[m, n]$, the probability that there is an impulse noise is $q$ and probability is $1-q$ that there is no impulse noise. Intuitively, $q$ equals the noise ratio. In typical applications, the amplitude of impulse noise is assumed to be fixed or follow Gaussian or uniform distribution.

\section{B. Standard Median Filter and Types of Error}

Consider an image $\mathbf{S}$ and an observation $\mathbf{X}$ of size $M \times N$

$$
\mathbf{X}=\mathbf{S}+\mathbf{U}
$$

where $\mathbf{U}$ is the impulse noise. In the above model, noise is additive to the signal. In simulation, clipping at pixel value 255 is applied to keep the simulated corrupted pixel value within the range from 0 to 255 , for an 8 -bit monochrome image. The median filter is applied over a window surrounding the current pixel $\mathbf{X}(m, n)$ as

$$
\begin{aligned}
\mathbf{Y}(m, n)= & \operatorname{MF}(\mathbf{X}(m, n), W) \\
= & \operatorname{median}\{\mathbf{X}(m-k, n-l),(k, l) \in W\}, \\
& m=1, \ldots, M, \quad n=1, \ldots, N
\end{aligned}
$$

where $W$ is a predetermined window. Usually, $W$ is chosen to be $3 \times 3,5 \times 5$, or $7 \times 7$ [2].

In detecting and removing impulse noise, the median filter makes can make a number of different types of mistakes. Type I errors (also known as misses [19]) occur when there is noise corrpting a pixel but the median filter does not detect it. False alarms, Type II errors happen when the median filter detects an impulse noise when there is actually no noise. For example, assuming a signal is given by

$$
\mathbf{S}=\left[\begin{array}{lllll}
1 & 5 & 5 & 5 & 1 \\
1 & 1 & 5 & 1 & 1 \\
1 & 1 & 5 & 1 & 1 \\
1 & 1 & 5 & 1 & 1 \\
1 & 5 & 5 & 5 & 1
\end{array}\right]
$$

and there is no noise, at position $(3,3)$, the $3 \times 3$ median filter will generate the result (here we assume that at the boundaries, the median filter symmetrically extends $\mathbf{S}$ before finding the median value in the filter window)

$$
\mathbf{Y}=\left[\begin{array}{lllll}
1 & 5 & 5 & 5 & 1 \\
1 & 1 & 5 & 1 & 1 \\
1 & 1 & 1 & 1 & 1 \\
1 & 1 & 5 & 1 & 1 \\
1 & 5 & 5 & 5 & 1
\end{array}\right]
$$

which is a Type II error.

Additionally, the median filter is also prone to a third type of difficulty, which we call over-correcting and label it Type III error. This type of error happens when there is impulse noise of low amplitude and the median filter removes the impulse noise and replaces it with the median value of in the filter window. When the median value is not as close to the true pixel value as the noisy pixel is, an over-correcting error occurs. Using the above signal for example and assuming there is a low amplitude impulse noise such as

$$
\mathbf{U}=\left[\begin{array}{lllll}
0 & 0 & 0 & 0 & 0 \\
0 & 0 & 0 & 0 & 0 \\
0 & 0 & 2 & 0 & 0 \\
0 & 0 & 0 & 0 & 0 \\
0 & 0 & 0 & 0 & 0
\end{array}\right]
$$

then the observation will be

$$
\mathbf{X}=\left[\begin{array}{lllll}
1 & 5 & 5 & 5 & 1 \\
1 & 1 & 5 & 1 & 1 \\
1 & 1 & 7 & 1 & 1 \\
1 & 1 & 5 & 1 & 1 \\
1 & 5 & 5 & 5 & 1
\end{array}\right]
$$

To find the correct value at position $(3,3)$, the $3 \times 3$ median filter will generate a result of

$$
\mathbf{Y}=\left[\begin{array}{lllll}
1 & 5 & 5 & 5 & 1 \\
1 & 1 & 5 & 1 & 1 \\
1 & 1 & 1 & 1 & 1 \\
1 & 1 & 5 & 1 & 1 \\
1 & 5 & 5 & 5 & 1
\end{array}\right]
$$

instead of the original signal. In this case, the output is incorrect and moreover, the output of median filter has a larger error than the original noisy observation. Therefore, it is beneficial to adaptively choose when to keep median filter output and when to keep the original pixel value. 
Step 1. For each pixel $\mathbf{X}(m, n)$, applying median filter

$$
\mathbf{Y}(m, n)=\mathcal{M F}\left(\mathbf{X}(m, n), W_{1}\right)
$$

and generates an error index matrix

$$
\mathbf{E}_{1}=\Omega(\mathbf{X}-\mathbf{Y}) .
$$

Step 2. Adaptively replace some original pixels of $\mathbf{Y}$ by original pixels of $\mathbf{X}$

$$
\begin{aligned}
\tilde{\mathbf{Y}} & =\Psi\left(\mathbf{Y}, \mathbf{E}_{1}\right) \\
\mathbf{E}_{2} & =\Omega(\mathbf{Y}-\tilde{\mathbf{Y}})
\end{aligned}
$$

where $\Psi$ represents the adaptive processor shown in Fig. 2.

Step 3. For each pixel $\tilde{\mathbf{Y}}(m, n)$

$$
\mathbf{Z}(m, n)=\left\{\begin{array}{cc}
\mathcal{M} \mathcal{F}\left(\tilde{\mathbf{Y}}(m, n), W_{2}\right), & \text { if } \mathbf{E}_{2}(m, n)=0 \\
\mathbf{X}(m, n), & \text { if } \mathbf{E}_{2}(m, n)=1
\end{array}\right.
$$

Fig. 1. Steps of adaptive method.

$$
\begin{aligned}
& \text { 2-a Choose parameter } \eta=a \sigma_{\lambda} \text { where } a>0 \text { and } b>0 \\
& \text { 2-b FOR } n=1: N \\
& \text { - } \lambda(n)=\frac{\sum_{m=1}^{M} \mathbf{E}_{1}(m, n)}{M} \\
& \text { - } \operatorname{IF}\left(\lambda(n)-\mu_{\lambda}\right)>\eta \\
& \text { - Let } \\
& \mathbf{e}=\mathbf{X}(:, n)-\mathbf{Y}(:, n) \\
& \text { - Let } K=\operatorname{round}\left(\lambda(n)-\mu_{\lambda}+b \sigma_{\lambda}\right) \\
& -\mathbf{v}=\Gamma(\mathbf{e}, K) \\
& \tilde{\mathbf{Y}}(m, n)= \begin{cases}\mathbf{X}(m, n) & \text { for } m \in \mathbf{v}, m=1, \ldots, M \\
\mathbf{Y}(m, n) & \text { otherwise }\end{cases}
\end{aligned}
$$

2-c ENDFOR

Fig. 2. Adaptive processor $\Psi$ to detect impulse noise.

\section{ATPMF to Detect Impulse Noise}

To improve the performance of the median filter, we introduce an ATPMF. The idea here is to use the first pass of the median filter to clean up the image and obtain an estimate of the spatial distribution and amplitude of the impulse noise. Using the estimated impulse noise, an adaptive process is carried out to selectively replace some pixels by the original pixel values of $\mathbf{X}$. These pixels are kept unchanged in the second median filtering, which is used to remove additional noise.

To facilitate the discussion, we introduce two operators. The first operator $\Omega$ takes a vector or matrix input and marks nonzero elements of the input by one and assigns zeros to other elements

$$
\Omega(x)= \begin{cases}1, & \text { if } x \neq 0 \\ 0, & \text { if } x=0\end{cases}
$$

For example

$$
\Omega\left(\left[\begin{array}{lll}
1 & 0 & 3 \\
0 & 5 & 2 \\
3 & 0 & 0
\end{array}\right]\right)=\left[\begin{array}{lll}
1 & 0 & 1 \\
0 & 1 & 1 \\
1 & 0 & 0
\end{array}\right]
$$

The second operator $\Gamma$ takes a vector $\mathbf{u}=\left[u_{1}, \ldots, u_{N}\right]^{T}$, where $T$ denotes the transpose and returns the positions of the first $k$ smallest elements of $\mathbf{u}$

$$
\mathbf{v}_{k \times 1}=\Gamma(\mathbf{u}, k), \quad k=1, \ldots, N
$$

For example, if $\mathbf{u}=[2,5,1,3,6,4,7]^{T}$, then

$$
\Gamma(\mathbf{u}, 3)=[3,1,4]^{T} \text {. }
$$

We now describe the three steps of our algorithm-see Fig. 1. Step 1 is the standard median filtering, (5). The matrix $\mathbf{E}_{1}$, which we call the error index matrix (EIM), records pixel positions of $\mathbf{Y}$ different from $\mathbf{X}$. Pixels at these positions are supposedly contaminated by impulse noise. Step 2 analyzes $\mathbf{Y}$ and $\mathbf{E}_{1}$ to determine which pixels are most likely over-corrected by the first median filter. The over-corrected pixels are then replaced by their original values and kept unchanged in the third step. Step 2 also generates the second error index matrix $\mathbf{E}_{2}$ which determines which pixels remain unchanged in Step 3. Step 3 carries out the second pass of filtering wherever $\mathbf{E}_{2}(m, n)=0$.

Details of Step 2 are given in Fig. 2. Here, the notation $\mathbf{A}(:, n)$ stands for the $n$th column of a matrix $\mathbf{A}$. In Step 2-a, 


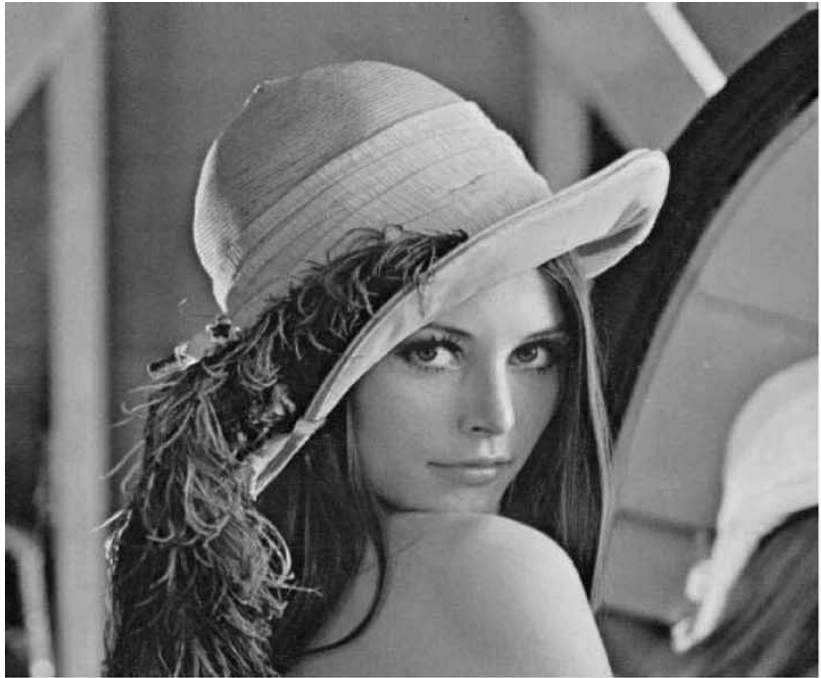

(a)

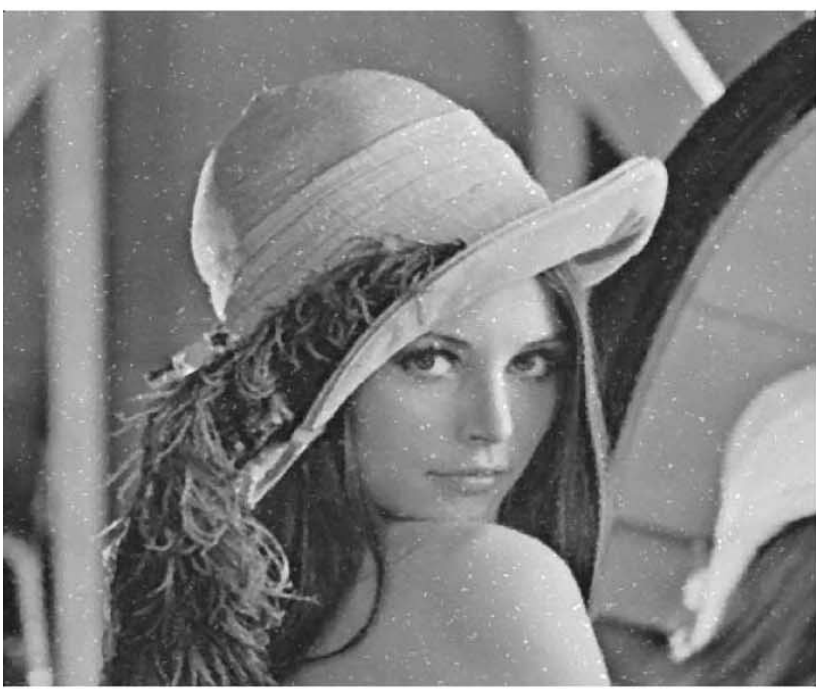

(c)

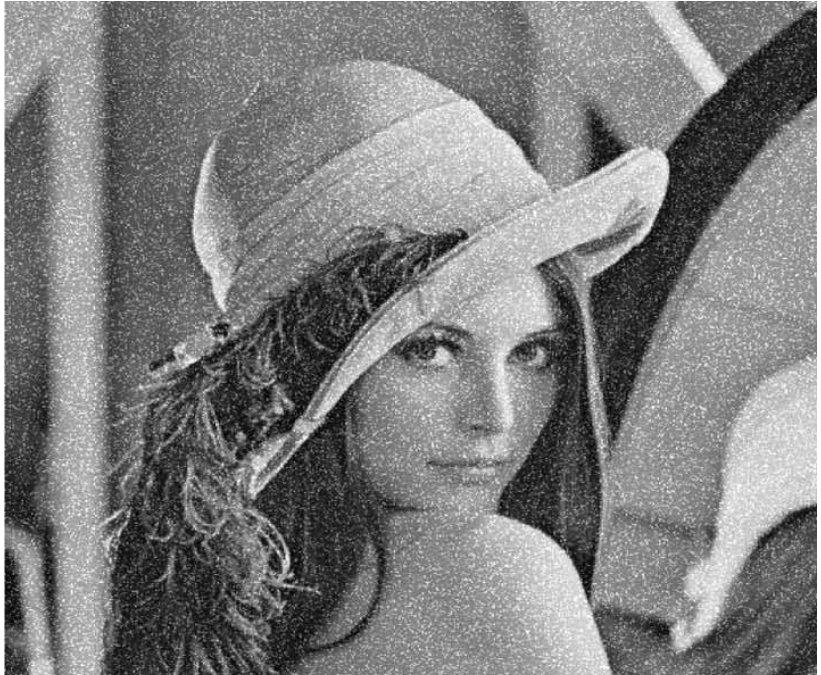

(b)

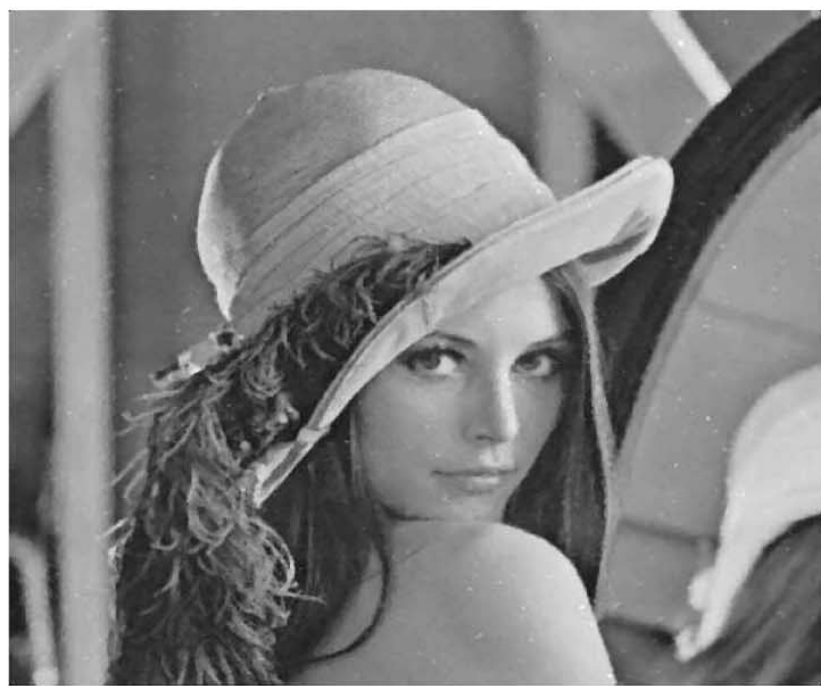

(d)

Fig. 3. Standard median filter to remove impulse noise: (a) original image; (b) noisy observation; (c) restored image by the $3 \times 3$ median filter; and (d) restored image by the ATPMF. Noise ratio is $25 \%$. Impulse noise has a Gaussian distribution with mean 30 and standard deviation of 5 .

predetermined parameter $a$ controls the threshold of detecting a column over-corrected and $b$ controls how many pixels will be replaced by their original values. Step 2-b and 2-c loop over subimages to remedy over-correction. In Fig. 2, we apply the ATPMF by column. It can be easily modified to proceed by row. In Step 2, the columnwise noise ratio is first estimated as

$$
\lambda(n)=\frac{\sum_{m=1}^{M} \mathbf{E}_{1}(m, n)}{M}, \quad n=1, \ldots, N .
$$

Second, we compute the mean $\mu_{\lambda}$ and standard deviation $\sigma_{\lambda}$ of $\lambda(n)$. For an impulse noise satisfying a binary distribution, by the De Moivre-Laplace theorem [20], as $M$ goes large, $\lambda(n)$ will approximate a Gaussian distribution $N\left(\mu_{\lambda}, \sigma_{\lambda}\right)$ where $\mu_{\lambda}=$ $M p$ and $\sigma_{\lambda}=M p(1-p)$.

Before moving on, we note that because Step 2 of our approach operates only on those pixels identified in the first step as contaminated, it is in fact independent of the filter used in Step 1. Therefore, we can replace the standard median filter in Step 1 by any other rank order filter, as we will show in next section.

Fig. 3(a)-(c) shows a true image, a noisy observation $\mathbf{X}$, and the restored image by the $3 \times 3$ median filter. The impulse noise has a Gaussian distribution in amplitude and a noise ratio of 25\%. Fig. 3(d) shows the result obtained by ATPMF. It is seen that the ATPMF produces a cleaner image than the standard median filter.

Fig. 4(a) plots $\lambda(n)$ using the normplot command of the MATLAB, for a true sample of impulse noise. It is seen that the curve matches a Gaussian distribution very well. Fig. 4(b) plots the estimated $\lambda(n)$ for the example shown in Fig. 3. It is seen that the estimated number of noisy pixels per column is close to a Gaussian distribution. Therefore when $\lambda(n)$ differs too much from $\mu_{\lambda}$, it is believed that some pixels of column $n$ are 


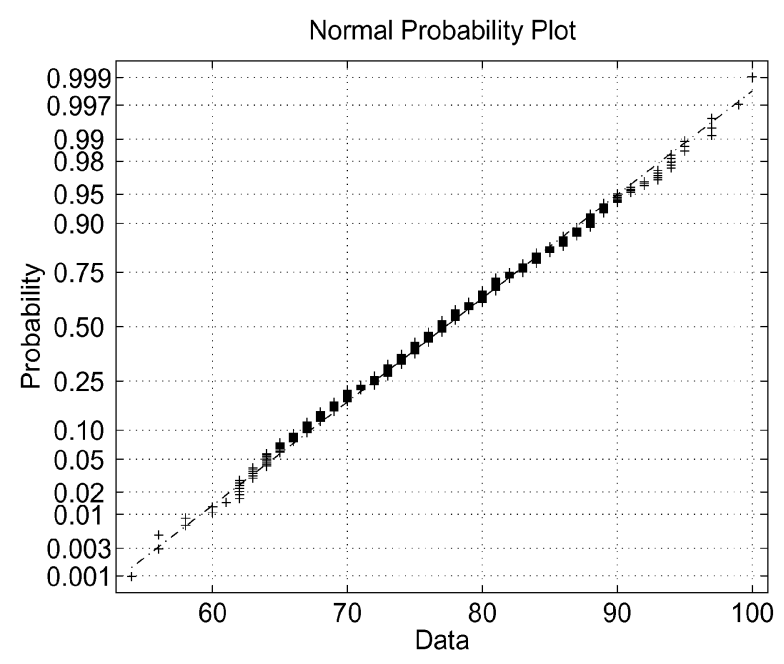

(a)

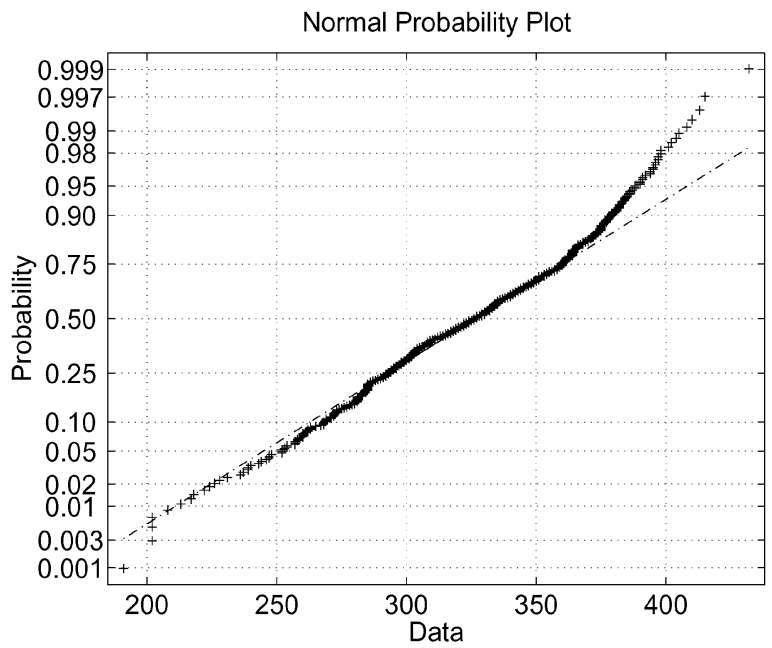

(b)

Fig. 4. (a) True columnwise distribution of noisy pixels. (b) Estimated columnwise distribution of noisy pixels by the $3 \times 3$ median filter. Horizontal axis: number of noisy pixels in a column. Vertical axis is the probability of a column of such number of noisy pixels. A straight line of "+" represents a Gaussian distribution.

over-corrected by the first median filter and should be replaced by the original pixels. Using $\mu_{\lambda}$ and $\sigma_{\lambda}$, at each column we make a one-side parameter test

$$
\begin{aligned}
& \mathcal{H}_{0}: \lambda(n) \sim N\left(\mu_{\lambda}, \sigma_{\lambda}^{2}\right) \\
& \mathcal{H}_{1}: \lambda(n) \sim N\left(\mu_{\lambda}+A, \sigma_{\lambda}^{2}\right)
\end{aligned}
$$

or equivalently (after subtracting $\mu_{\lambda}$ from the observation)

$$
\begin{aligned}
& \mathcal{H}_{0}: \lambda(n) \sim N\left(0, \sigma_{\lambda}^{2}\right) \\
& \mathcal{H}_{1}: \lambda(n) \sim N\left(A, \sigma_{\lambda}^{2}\right)
\end{aligned}
$$

where $A>0$ and is unknown, to detect columns containing unlikely large number of impulse noise. The null hypothesis indicates that the number of pixels containing impulse noise in the $n$th column is reasonably small and most of those pixels altered by the median filter truly contain impulse noise. The alternative hypothesis represents the case that there is a excessively large number of pixels altered by the median filter and therefore it is likely some of these pixels do not contain impulse noise and are mistakenly changed by median filtering. Although $A$ is unknown, knowing that $A>0$ allows us to find a uniformly most powerful (UMP) test [21] using the Neyman-Pearson criterion. The Neyman-Pearson test is to decide $\mathcal{H}_{1}$ if [19]

$$
\frac{p\left(\lambda ; \mathcal{H}_{1}\right)}{p\left(\lambda ; \mathcal{H}_{0}\right)}=\frac{\frac{1}{\sqrt{2 \pi} \sigma_{\lambda}} \exp \left[-\frac{(\lambda-A)^{2}}{2 \sigma_{\lambda}^{2}}\right]}{\frac{1}{\sqrt{2 \pi} \sigma_{\lambda}} \exp \left[-\frac{\lambda^{2}}{2 \sigma_{\lambda}^{2}}\right]}>\eta
$$

where $\eta$ is a preset threshold. Taking the logarithm of and simplifying (22), we have

$$
A \lambda>\sigma_{\lambda}^{2} \ln \eta+\frac{A^{2}}{2} .
$$

Since $A>0$, we then have

$$
\lambda>\frac{\sigma_{\lambda}^{2}}{A} \ln \eta+\frac{A}{2} \equiv \eta^{\prime} .
$$

The probability of false-alarm is

$$
P_{F A}=Q\left(\frac{\eta^{\prime}}{\sigma_{\lambda}}\right)
$$

where $\eta^{\prime}$ can be decided by pre-selecting the $P_{F A}$ and $Q$ is the complementary cumulative distribution function of a standard Gaussian probability density function. Then solving the following equation gives $\eta^{\prime}$ :

$$
\eta^{\prime}=\sigma_{\lambda} Q^{-1}\left(P_{F A}\right)
$$

which is independent of $A$. After finding $\eta^{\prime}$ from (26), the probability of detection is given by

$$
P_{D}=Q\left(\frac{\eta^{\prime}-A}{\sigma_{\lambda}}\right) \text {. }
$$

The above test is UMP in the sense that for a fixed $P_{F A}$, it yields the highest $P_{D}$ among all the tests [21]. As expected, increasing $\eta$ (or $\eta^{\prime}$ ) reduces both $P_{F A}$ and $P_{D}$, and vice versa. In Section III, we investigate the effect of different $\eta$ on the performance of the ATPMF.

\section{Choice of $a$ and $b$ in Step-2}

In Step-2, $a$ and $b$ can be chosen based on how tight the Gaussian distribution in columnwise noise ratio shall be. In other words, we can choose how much deviation is acceptable between the $\lambda(n)$ and $\mu_{\lambda}$ before we declare there is over-correction in column $n$. For example, we can choose $a=1$ so that if a columnwise noise ratio deviates from the mean value by one standard deviation, we will consider that column being over-corrected by the first filtering. Similarly, we can set $b=1$ to correct that many pixels in the column.

\section{E. Different Implementations}

In the above algorithm, the adaptive process is carried out column by column. On the other hand, the adaptive process can be implemented by rows. Fig. 5 shows the restored images of 


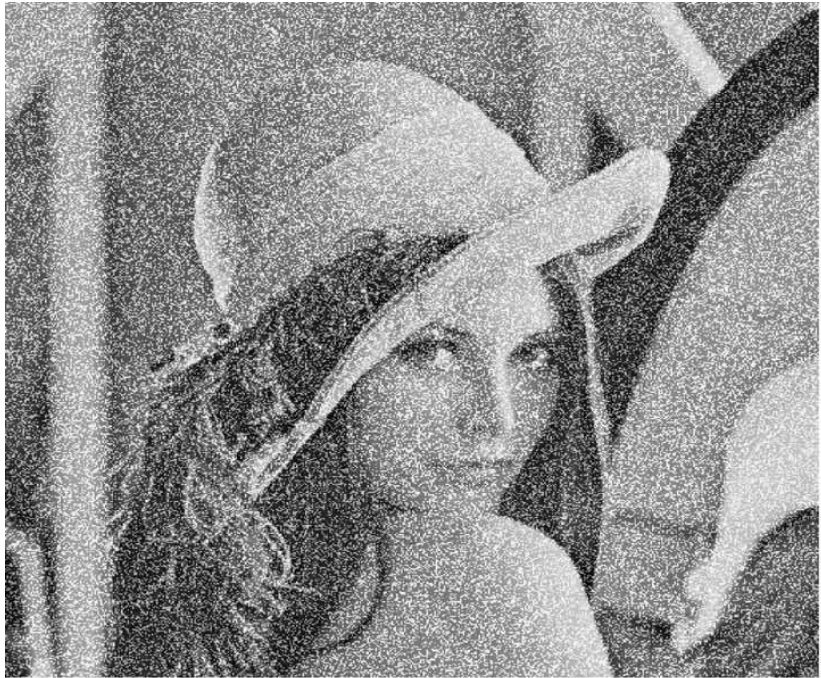

(a)

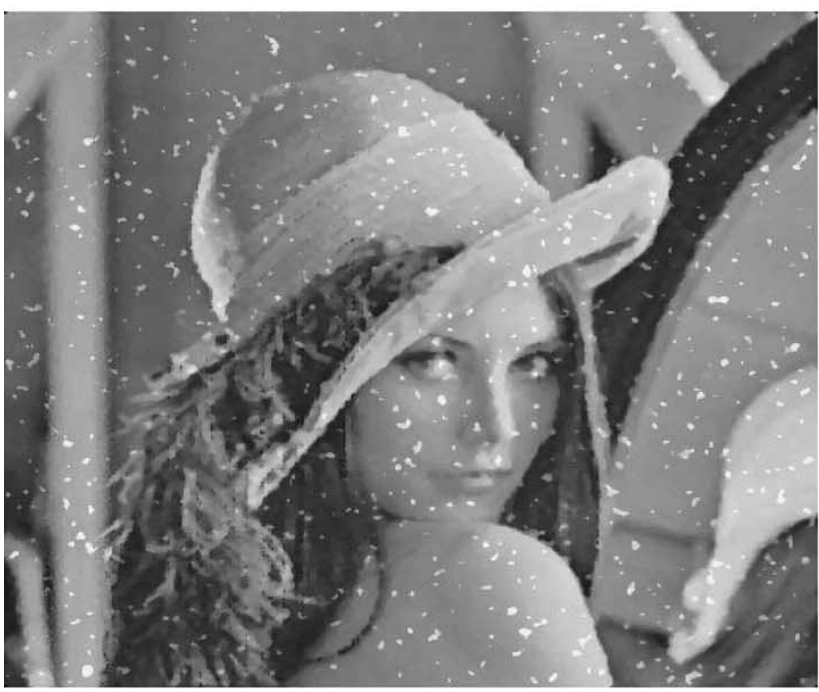

(c)

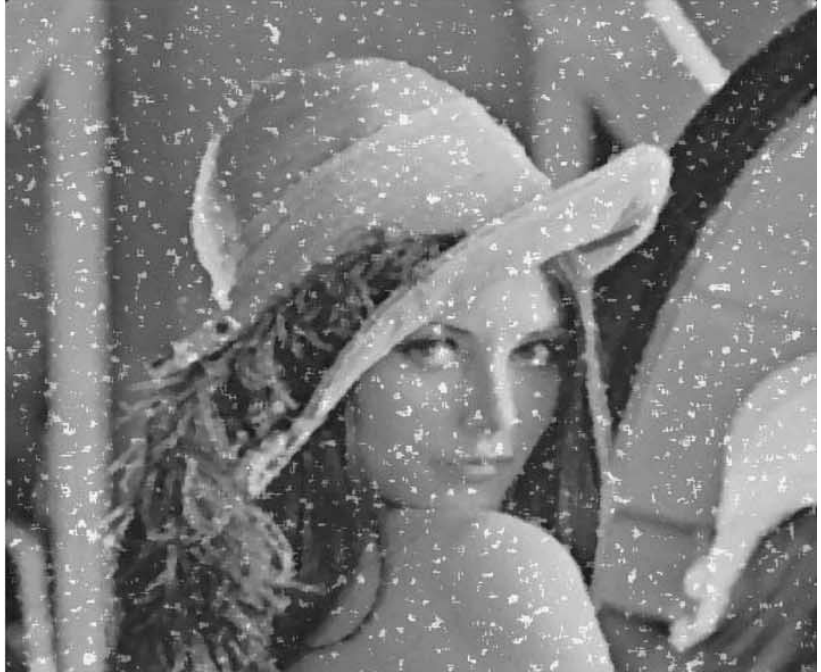

(b)

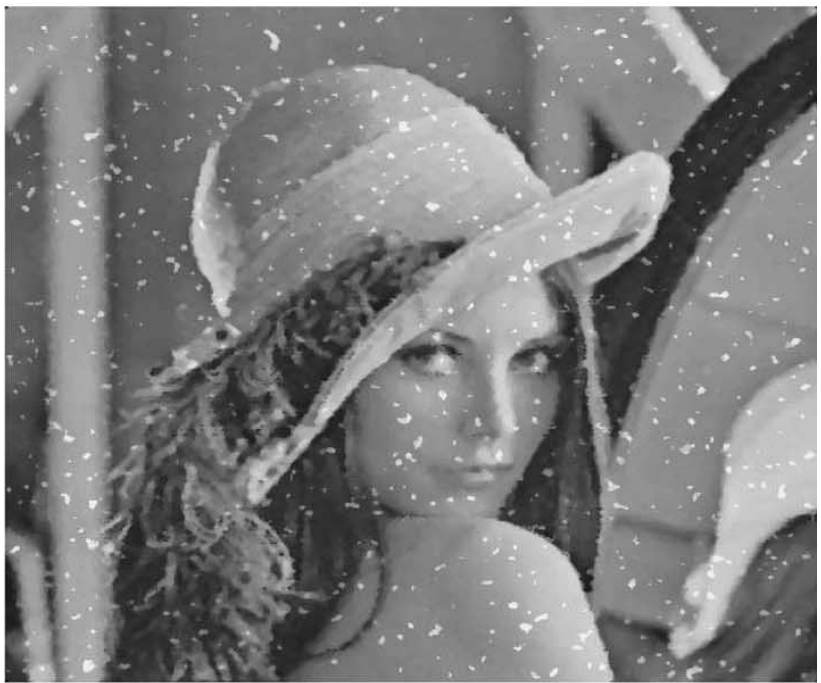

(d)

Fig. 5. Different implementation. (a) Noisy observation of "Lena," noise ratio is $35 \%$. (b) Restored image by a standard $5 \times 5$ filter, PSNR $=20.8517$ (dB). (c) Restored image by ATPMF implemented in columns, PSNR $=22.8672$ (dB). (d) Restored image by ATPMF implemented in rows, PSNR $=22.7692(\mathrm{~dB})$

"Lena" by the $5 \times 5$ median filter and by an ATPMF implemented in columns and in rows. For the adaptive two-pass filter, we set $a=1$ and $b=1$. Comparing the two images of ATPMF, we see there is little difference between them, yet they both are much better than the standard median filter output.

\section{F. Computational Issue}

Because of the increased steps of computation, the computational time of applying adaptive two-pass filtering is longer than one-pass filtering. The most time-consuming part of our method is to run the underlying rank order based filter. The adaptive part of our method, i.e., Step 2 in Fig. 1, takes much less time to complete. Depending on the underlying filter used, step 2 takes about $3 \%$ to $5 \%$ of the total computational time.

\section{EXAMPLES}

In this section, we use some examples to demonstrate the performance of our algorithm and generalize the idea of our

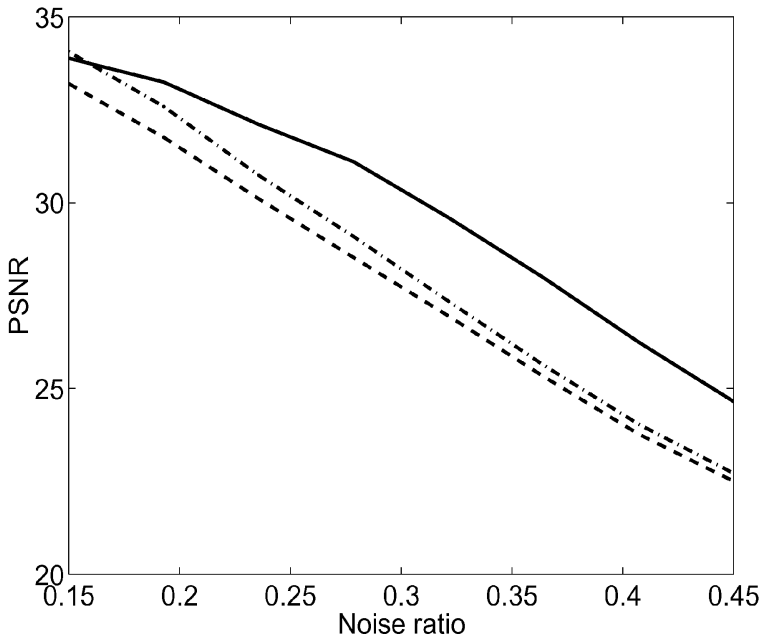

Fig. 6. PSNR of "Lena." Dashed line: $3 \times 3$ median filter. Dashed-dotted line: two-pass $3 \times 3$ median filter. Solid line: ATPMF. For the ATPMF, the underlying median filter window is $3 \times 3$ and $a=1, b=1$. 


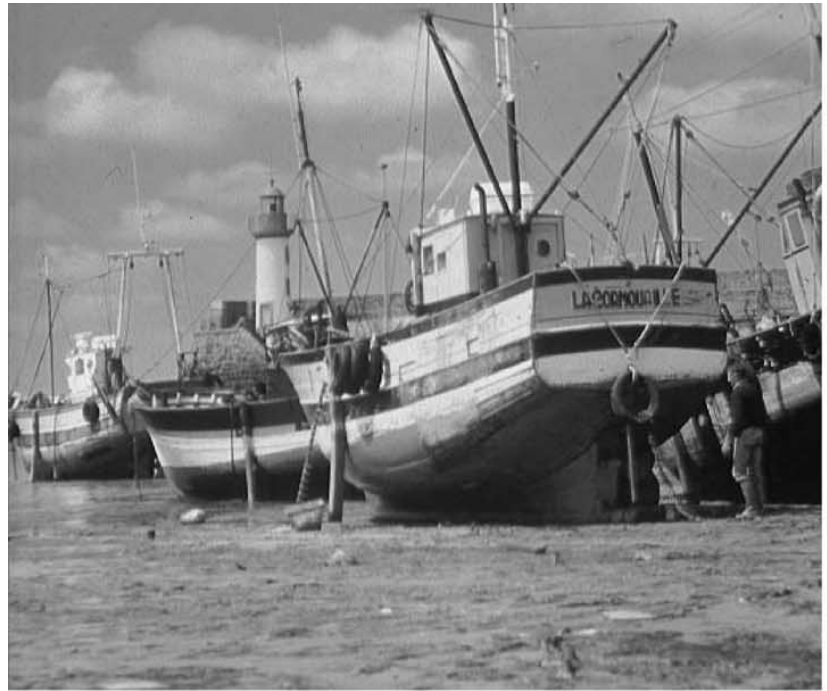

(a)

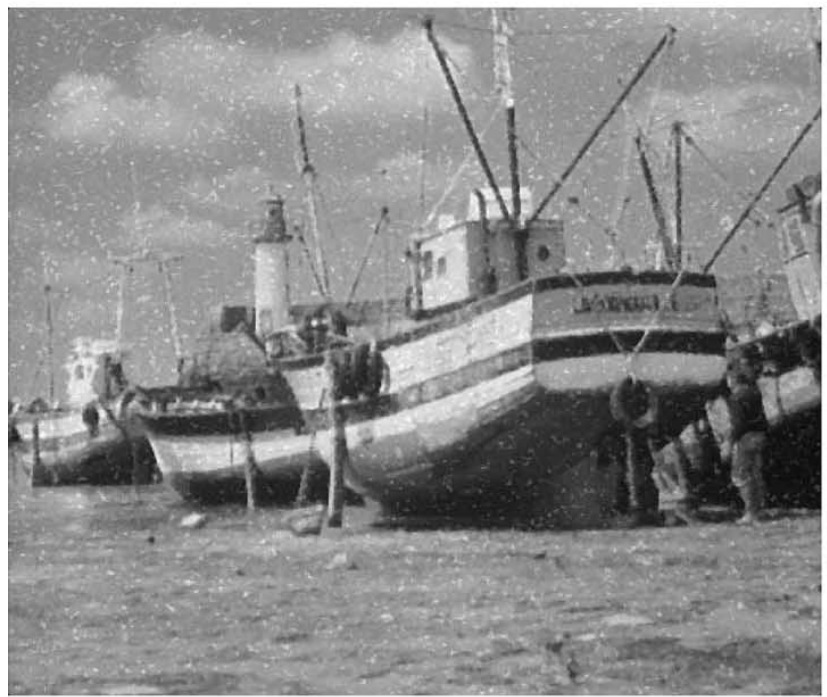

(c)

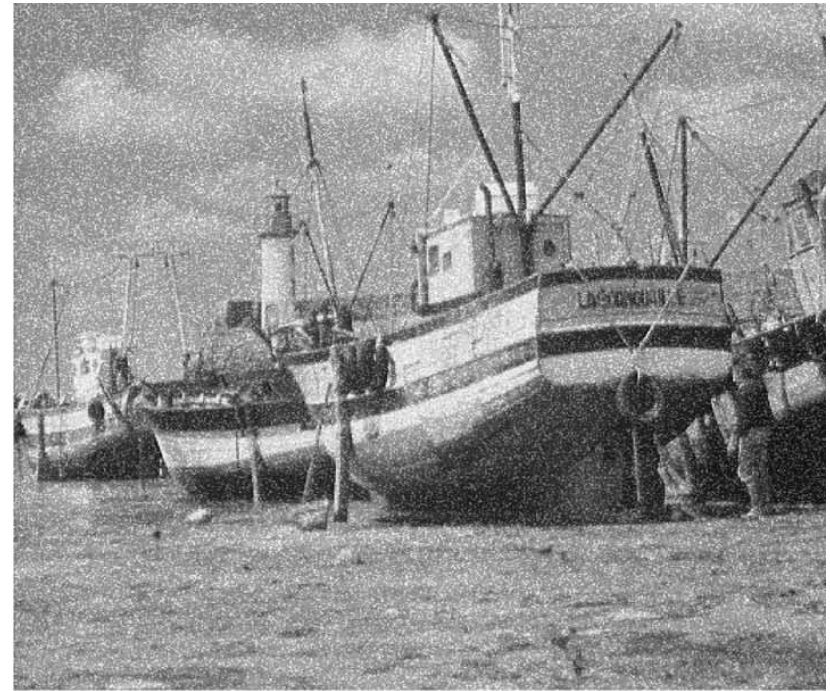

(b)

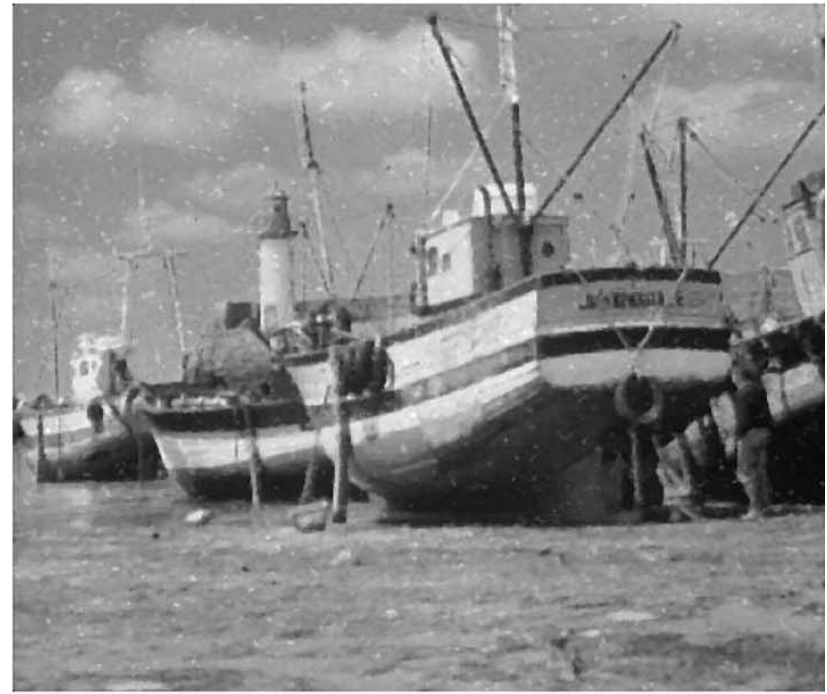

(d)

Fig. 7. Median filter to remove impulse noise. (a) Original image. (b) Noisy observation, noise ratio is $30 \%$. (c) Restored image by the $3 \times 3$ median filter, PSNR $=24.3833(\mathrm{~dB})$. (d) Restored image by the ATPMF, PSNR $=25.8376(\mathrm{~dB})$. Adaptive process is implemented in columns with $a=1, b=1$.

method to other rank-order-based filters such as CWMF, adaptive weighted median filter, lower-upper-middle (LUM) filter, and SD-ROM filter. Quantitatively, we use PSNR to compare the restored images with and without using the adaptive process. For a final output image $\mathbf{Z}$, the PSNR is defined as

$$
\operatorname{PSNR}=10 \log _{10} \sum_{m=1}^{M} \sum_{n=1}^{N} \frac{255^{2}}{(\mathbf{Z}(m, n)-\mathbf{S}(m, n))^{2}}(\mathrm{~dB}) .
$$

\section{A. Detect Impulse Noise Based on Median Filter}

Fig. 6 compares the PSNR of the standard median filter, the two-pass median filter and the ATPMF obtained in processing the "Lena" image. At high noise ratios, the ATPMF results have much higher PSNR than those of the standard median filter. It is interesting to observe that by simple two-pass median filtering, results become modestly better. By introducing the adaptive process between two median filters, much improved PSNRs are obtained at all noise ratios, especially above $30 \%$. In the ATPMF, $a$ and $b$ are set to 1 . For the second example, we use the "boat" image. Fig. 7 shows the original image, a noisy observation at a ratio noise of $30 \%$, restored images by the $3 \times$ 3 median filter and the ATPMF. Fig. 8 shows the result of processing the "boat" image contaminated by impulse noise with a uniform distribution in amplitude.

\section{B. Effect of $\eta$ on the ATPMF Performance}

In the above section, we showed that by modeling the detection problem as a one side parameter hypothesis testing, we can find a UMP test based on Neyman-Pearson criterion. Here, we investigate the effect of $\eta$ on the performance of the ATPMF. Fig. 9 plots the PSNR of the "Lena" image restored by a two-pass median filter and the ATPMF for different $\eta$. As $\eta$ increases, PSNR increases at first and then decreases. As a threshold, $\eta$ controls how many "abnormal" noisy pixels will 


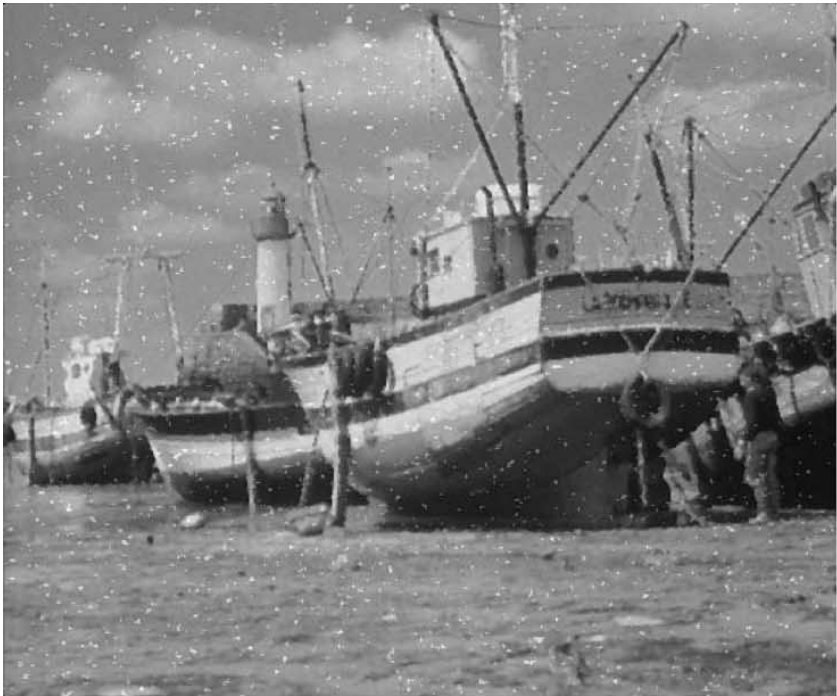

(a)

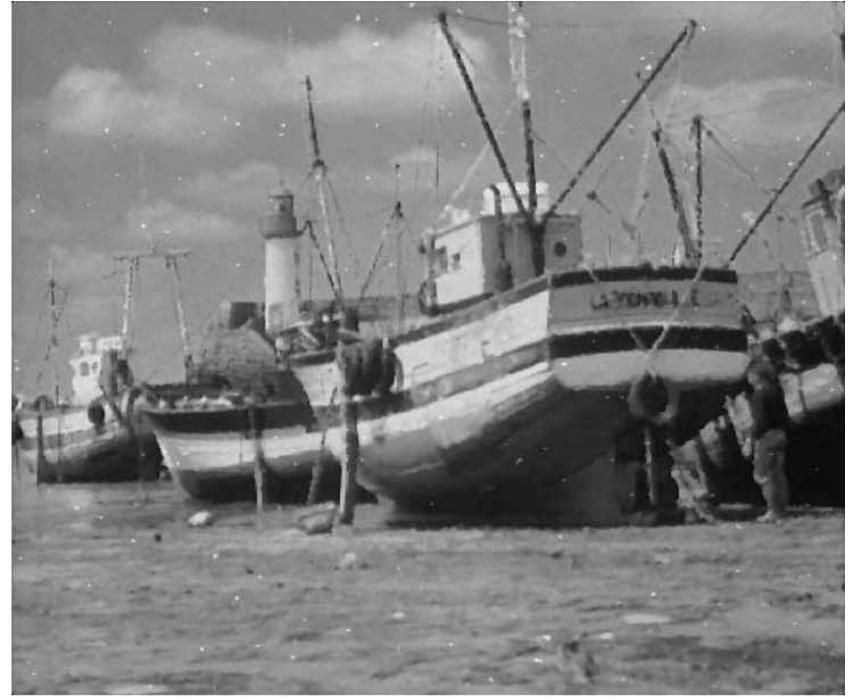

(b)

Fig. 8. Restored "boat" image by: (a) the $3 \times 3$ median filter, PSNR $=25.0612$ (dB) and (b) an ATPMF implemented in rows, $a=1, b=1$, PSNR $=$ $27.7221(\mathrm{~dB})$. Noise ratio is $20 \%$. Noise has a uniform distribution between 0 and 80 .

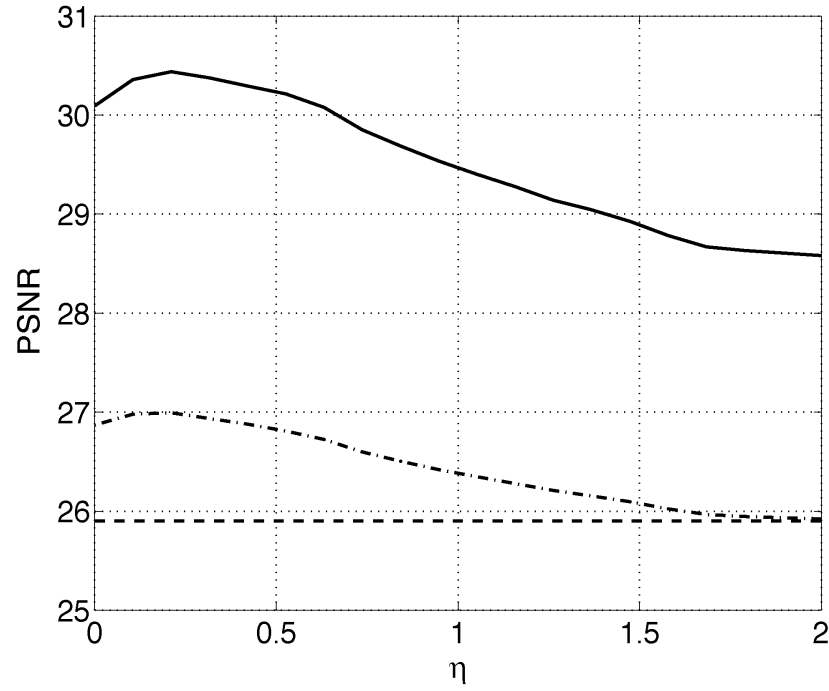

Fig. 9. PSNR of "Lena" for different $\eta$. Dashed line: standard median filter Dashed-dotted line: two-pass median filter. Solid line: ATPMF. Note that the standard median filter is not affected by the choice of $\eta$, and therefore its plot is a straight line. The noise ratio is $25 \%$ and noise has a Gaussian distribution for the amplitude.

be detected. An $\eta$ too small will declare too many noisy pixels "abnormal" and have the risk of over-correcting the results following the first pass of the filter. A large $\eta$ raises the threshold and detects a small number of "abnormal" noisy pixels and therefore have reduced improvement in performance. While a proper $\eta$ can be chosen based on prior information about the hardware, the noise ratio, and the type of image to be processed, we note that for this example at least, the performance of the ATPMF for any reasonable $\eta$ is significantly better than a standard median filter.

\section{Generalization to Other Rank Order Filters}

In recent years, many algorithms have been proposed to reduce impulse noise. Some of them are CWMF [10], [11], [17], adaptive center weighted median filter (ACWMF) [13], LUM filter [22], and SD-ROM filter [15]. In this section, we apply our algorithm to these filters and show that improved results are obtained in each case, respectively.

Table I shows the results of removing impulse noise with a random amplitude satisfying a Gaussian distribution. In the table, the second row shows the PSNR of using the standard 3 $\times 3$ median filter (MF), CWMF with a filter window of $3 \times 3$ and a center weight of 5, ACWMF, LUM filter of $(5,5,13)$ [22], and SD-ROM. The third row shows the results of applying our algorithm based on the corresponding filter. For all the examples, we set $a=1$ and $b=1$ in the adaptive process. It is seen that our algorithm improves the result of each filter, respectively. As expected, the better the performance of the underlying rank order filter, the better the results of the adaptive two-pass counterpart.

Table II shows the results of processing for an impulse noise with a uniform distribution between 0 and 120 while Table III summarizes the performance when the amplitude value is fixed at 100 . From both tables we can see that our algorithm improves the performance of the underlying rank order filters.

\section{Processing Salt and Pepper Noise}

Rank order filters are very effective in removing salt and pepper noise. In our algorithm, the adaptive part can be easily modified to remove salt and pepper noise by change (18) in Step-2 to

$$
\mathbf{e}=|\mathbf{X}(:, n)-\mathbf{Y}(:, n)| .
$$

Table IV shows the result of removing salt and pepper noise from the image of "Lena" using different rank order filters. As in the previous example, the addition of a second pass and adaptivity in the filtering leads to improvements in performance. 
TABLE I

IMAGE OF “LENA," NOISE RATIO Is 30\%, GAUSSIAN DistRIBUTION IN AMPLITUDE

\begin{tabular}{l||c|c|c|c|c}
\hline & MF & CWMF & ACWMF & LUM & SD-ROM \\
\hline \hline PSNR (dB) (regular) & 20.9160 & 22.8093 & 23.2441 & 20.9670 & 26.2251 \\
\hline PSNR (dB) (adaptive) & 24.6247 & 25.7156 & 26.4832 & 25.2988 & 28.5644 \\
\hline
\end{tabular}

TABLE II

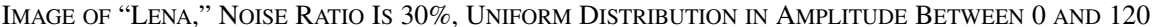

\begin{tabular}{l||c|c|c|c|c}
\hline & MF & CWMF & ACWMF & LUM & SD-ROM \\
\hline \hline PSNR (dB) (regular) & 26.5685 & 28.0796 & 28.6759 & 26.9210 & 27.7217 \\
\hline PSNR (dB) (adaptive) & 29.6517 & 29.9542 & 30.8357 & 30.2643 & 31.4640 \\
\hline
\end{tabular}

TABLE III

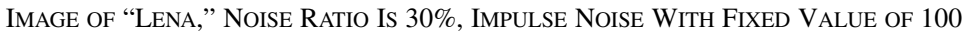

\begin{tabular}{l||c|c|c|c|c}
\hline & MF & CWMF & ACWMF & LUM & SD-ROM \\
\hline \hline PSNR (dB) (regular) & 21.5631 & 24.3777 & 23.0800 & 22.2382 & 29.0991 \\
\hline PSNR (dB) (adaptive) & 26.2769 & 27.7248 & 27.2482 & 26.3723 & 31.6330 \\
\hline
\end{tabular}

TABLE IV

Image of “Lena," Noise Ratio Is 30\%, SAlt \& PePPer Noise, Gaussian Distribution in Amplitude

\begin{tabular}{l||c|c|c|c|c}
\hline & MF & CWMF & AWMF & LUM & SD-ROM \\
\hline \hline PSNR (dB) (regular) & 36.2007 & 39.5893 & 39.8517 & 37.5196 & 37.5220 \\
\hline PSNR (dB) (adaptive) & 41.2992 & 41.9990 & 43.3003 & 41.7177 & 42.1703 \\
\hline
\end{tabular}

\section{CONCLUSION}

We presented an adaptive rank order filtering process to remove impulse noise in highly corrupted images. The adaptive filter is based on an underlying rank order filter such as the standard median filter, CWMF, adaptive weighted median filter, LUM filter, and SD-ROM filter. The adaptive process detects irregularities in the spatial distribution of the estimated impulse noise. By analyzing the first error index matrix, detection is accomplished using tools fro hypothesis testing theory. The method is able to correct some false alarms caused by the first filtering and remove remaining noise. We have shown that the adaptive filter performs better than using the underlying filter alone in removing impulse noise and reducing false alarms. Using test images and comparing the PSNR of the adaptive filter with those of the underlying filter, we demonstrated the improved performance of our method, especially at high noise ratios, on simulated images.

\section{REFERENCES}

[1] I. Pitas and A. N. Venetsanopoulos, Nonlinear Digital Filters: Principles and Applications. Norwell, MA: Kluwer, 1990.

[2] A. K. Jain, Fundamentals of Digital Image Processing. Englewood Cliffs, NJ: Prentice-Hall, 1989.

[3] G. R. Arce and R. E. Foster, "Detail-preserving ranked-order based filters for image processing," IEEE Trans. Acoust., Speech, Signal Processing, vol. 37, no. 1, pp. 83-98, Jan. 1989.
[4] A. C. Bovik, T. S. Huang, and D. C. Munson, "Edge-sensitive image restoration using order-constrained least squares methods," IEEE Trans. Acoust., Speech, Signal Processing, vol. 33, pp. 1253-1263, Oct. 1985.

[5] T. A. Nodes and N. C. Gallagher Jr., "The output distribution of median type filters," IEEE Trans. Commun., vol. COM-32, pp. 532-541, May 1984.

[6] A. C. Bovik, "Streaking in median filtered images," IEEE Trans. Acoust., Speech, Signal Processing, vol. 35, pp. 493-503, Oct. 1985.

[7] D. A. F. Florencio and R. W. Shafer, "Decision-based median filter using local signal statistics," in Proc. SPIE Symp. Visual Communication and Image Processing, vol. 2308, Sept. 1994, pp. 268-275.

[8] T. Chen and H. Wu, "Adaptive impulse detection using center-weighted median filters," Signal Processing Lett., vol. 8, no. 1, pp. 1-3, Jan. 2001.

[9] R. Yang, L. Lin, M. Gabbouj, J. Astola, and Y. Neuvo, "Optimal weighted median filters under structural constraints," IEEE Trans. Signal Processing, vol. 43, pp. 591-604, Mar. 1995.

[10] T. Song, M. Gabbouj, and Y. Neuvo, "Center weighted median filters: some properties and applications in image processing," Signal Processing, vol. 35, no. 3, pp. 213-229, 1994.

[11] S.-J. Ko and Y.-H. Lee, "Center weighted median filters and their applications to image enhancement," IEEE Trans. Circuits Syst., vol. 38, pp. 984-993, Sept. 1991.

[12] T. Sun and Y. Neuvo, "Detail-preserving median based filters in image processing," Pattern Recognit. Lett., vol. 15, no. 4, pp. 341-347, Apr. 1994.

[13] E. Abreu, M. Lightstone, S. K. Mitra, and K. Arakawa, "A new efficient approach for the removal of impulse noise from highly corrupted images," IEEE Trans. Image Processing, vol. 5, pp. 1012-1025, June 1996.

[14] Z. Wang and D. Zhang, "Progressive switching median filter for removal of impulsive noise from highly corrupted image," IEEE Trans. Circuits Syst. II, vol. 46, pp. 78-80, Jan. 1999. 
[15] H.-L. Eng and K.-K. Ma, "Noise adaptive soft-switching median filter," IEEE Trans. Image Processing, vol. 10, pp. 242-251, Feb. 2001.

[16] E. S. Hore, B. Qiu, and H. R. Wu, "Prediction based image restoration using a multiple window configuration," Opt. Eng., vol. 41, no. 8, pp. 1855-1865, Aug. 2002.

[17] L. Yin, R. Yang, M. Gabbouj, and Y. Neuvo, "Weighted median filters: A tutorial," IEEE Trans. Circuits Syst. II, vol. 43, pp. 157-192, Mar. 1996.

[18] H. G. Senel, R. A. Peters II, and B. Dawant, "Topological median filters," IEEE Trans. Image Processing, vol. 11, pp. 89-104, Feb. 2002.

[19] C. W. Helstrom, Elements of Signal Detection and Estimation. Englewood Cliffs, NJ: Prentice-Hall, 1995.

[20] S. C. Port, Theoretical Probability for Applications. New York: Wiley, 1994.

[21] S. M. Kay, Fundamentals of Statistical Signal Processing: Detection Theory. Englewood Cliffs, NJ: Prentice-Hall, 1993.

[22] R. C. Hardi and C. G. Boncelet, "LUM filters: a class of rank-orderbased filters for smoothing and sharpening," IEEE Trans. Signal Processing, vol. 41, pp. 1061-1076, Mar. 1993.

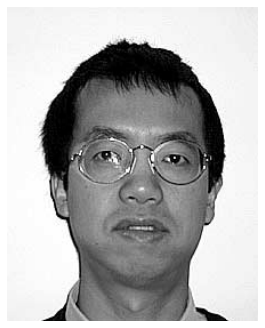

Xiaoyin Xu (S'99-M'03) received the M.S. an $\mathrm{Ph} . \mathrm{D}$. degrees in electrical engineering in 1998 and 2002, respectively, from Northeastern University, Boston, MA.

His research interests include subsurface object detection, image processing in remote sensing, statistic and adaptive signal processing, GPR and GPS signal processing, and nonlinear signal processing.
Eric L. Miller (S'90-M'95-SM '03) received the S.B. degree in 1990, the S.M. degree in 1992, and the Ph.D. degree in 1994, all in electrical engineering and computer science from the Massachusetts Institute of Technology, Cambridge.

$\mathrm{He}$ is currently an Associate Professor in the Department of Electrical and Computer Engineering, Northeastern University, Boston, MA. His research interests include physics-based tomographic image formation and object characterization, inverse problems in general and inverse scattering in particular, regularization, statistical signal and imaging processing, and computational physical modeling. This work has been carried out in the context of applications including medical imaging, nondestructive evaluation, environmental monitoring and remediation, landmine and unexploded ordnance remediation, and automatic target detection and classification.

Dr. Miller is currently serving as an Associate editor for the IEEE TRANSACtions on Geoscience and Remote SENSING and was in the same position at the IEEE TRANSACTIONS ON IMAGE PROCESSING from 1998-2002. He received the CAREER Award from the National Science Foundation in 1996 and the Outstanding Research Award from the College of Engineering at Northeastern University in 2002. He is a member of Tau Beta Pi, Phi Beta Kappa, and Eta Kappa Nu.

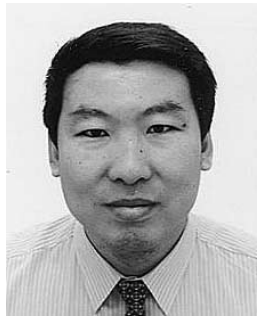

Dongbin Chen received the Ph.D. degree from the School of Computing and Technology, University of East London, London, U.K., in 2003.

He was a Senior Engineer with the Chinese Second Academy of Aero-Space from 1988 to 1998 . He is currently a Research Assistant with the Department of Design and Systems Engineering, Brunel University, Middlesex, U.K. His areas of research include image processing, image inspection, machine vision, computer vision, photogrammetry, and integration of vision application systems.

Mansoor Sarhadi received the Ph.D. degree in electronics from the University of London, London, U.K., in 1980.

He is Vice Principle and Professor in Engineering at Brunel University, Middlesex, U.K. His research interests include image processing, robotics, and automated manufacturing. He has published over 50 articles in his fields of research. Dr. Sarhadi is a member of IEE 